\title{
Die elektrochemische Messung des Sauerstoffgehaltes von Wasser, Abwasser und Schlamm
}

\author{
Von L. KalmaN, EAWAG, Zürich
}

\section{Messverfahren}

Die elektrochemische Messung des gelösten Sauerstoffes in Flüssigkeiten erfolgt auf der Grundlage des elektrochemischen Prinzips nach F. Tödt. Werden nämlich zwei verschiedene Metalle, z.B. Gold und Zink, in eine Elektrolytlösung getaucht, dann entsteht zwischen den Elektroden ein Depolarisationsstrom, dessen Grösse vom Gehalt an gelöstem Sauerstoff abhängig ist. Im wesentlichen wird dabei an der edleren Elektrode, d.h. an der Kathode, der Sauerstoff reduziert. Eine wesentliche Rolle spielt dabei die Geschwindigkeit, mit welcher der Sauerstoff durch die Grenzschicht der Flüssigkeit an der Elektrode diffundiert. Aus diesem Grunde ist der Strom abhängig von der Flüssigkeitsgeschwindigkeit. Das unedle Metall, also z. B. das Zink, geht dabei in Lösung und korrodiert um so stärker, je stärker der Strom ist.

Die Korrosion der Anode und der Verbrauch an Elektrolyt sind die Ursachen dafür, dass die mit Membran bedeckten Sauerstoffelektroden von Zeit zu Zeit revidiert und neu aufgefüllt werden müssen, und dass verhältnismässig häufige Nacheichungen notwendig sind. Auf der Membrane bildet sich bei langandauernden Messungen in verschmutzten Flüssigkeiten, wie z. B. im Abwasser und im Belebtschlamm, ein Belag, welcher die Diffusionsgeschwindigkeit des Sauerstoffs durch die Membrane und damit auch den Strom herabsetzt.

\section{Entwicklung einer neuen Sauerstoffsonde*}

Ziel der Entwicklung war, eine Saucrstoffelektrode zu schaffen, welche ohne Membrane arbeitet, bei welcher die aktiven Flächen sich im Laufe der Zeit nicht verändern, und welche längere Zeit ohne Nacheichung und Unterhalt in verschmutztem Wasser und im Belebtschlamm genau arbcitet. Der tatkräftigen Unterstützung der Professoren Jaag und Hörler ist es zu verdanken, dass eine derartige Sonde entwickelt werden konnte. Werden nun zwei verschiedene Metalle, wie z.B. Zink und Gold, ohne Membrane und Elektrolyt frei ins Leitungswasser getaucht, so wird man bald feststellen, dass der Verwendung als Sauerstoffsonde die folgenden Schwierigkeiten entgegenstehen, die die Anwendung als Sauerstoffsonde fraglich machen.

* Lizenznehmerin und serienmässige Fabrikation: Züllig, Mess- und Regelanlagen für die Wasserwirtschaft, CH-9424 Rheineck. 


\subsection{Korrosion und Kalkablagerung}

Ausser der schon erwähnten Korrosion der Zinkanode scheidet sich an der Kathode infolge des Stromes eine Kalkschicht aus, die den Strom nach einigen Stunden abklingen lässt.

\subsection{Einfluss der Geschreindigkeit}

Wie schon erwähnt, bewirkt die Zunahme der Fliessgeschwindigkeit des Wassers ein Ansteigen des elektrischen Stroms. Erst von einer Geschwindigkeit von etwa $1 \mathrm{~m} / \mathrm{sec}$ an ist die Anzeige unabhängig von der Geschwindigkeit.

Die bisher entwickelten Sauerstoffmessgeräte einschliesslich der Membranelektroden arbeiten alle bei diesem maximalen Strom und erfordern deshalb eine bestimmte Wassergeschwindigkeit, die entweder durch die natürliche Fliessgeschwindigkeit oder durch Pumpen erzeugt wird. Anders verhält es sich bei der neuen Sonde, wo bei einer in der Sonde selbst künstlich erzeugbaren mittleren Geschwindigkeit gemessen wird.

\subsection{Einfluss der Leitfähigkeit}

Eine weitere Schwierigkeit, die bei membranlosen Elektroden auftreten kann, ist der Einfluss des Elektrolytgehaltes. Mit steigender Leitfähigkeit steigt der Strom anfänglich sehr rasch, bei höherem Salzgehalt nur sehr langsam einem Maximum entgegen. $\mathrm{Da}$ wir in unseren Fliessgewässern und Abwässern mit schwankender Leitfähigkeit zu rechnen haben, ist es sehr wichtig, diesen Einfluss zu verkleinern. Bereits F. TöDт [1] und H. АмвӥнL [2] haben erkannt, dass sich durch Verkleinerung der edlen Kathodenfläche der Einfluss der Leitfähigkeit etwas vermindern lässt.

\section{Aufbau der Sonde}

Die Sonde besteht aus einem etwa 1,5 m langen Kunststoffrohr, welches mit einer leicht lösbaren Haltevorrichtung in einem Becken oder Kanal befestigt ist (Abb. 1). An dem unteren Ende des Rohres befindet sich der von der Eidg. Anstalt für Wasserversorgung, Abwasserreinigung und Gewässerschutz an der ETH Zürich (EAWAG) entwickelte und durch Patent geschützte Messkopf, der eine Reinigungsvorrichtung aufweist und so gestaltet ist, dass auch nach Abnutzung während eines Betriebes von mehreren Jahren die aktiven Flächen der Elektroden immer gleich gross bleiben, so dass keine spätere Nacheichung erforderlich ist. Eine spezielle Einrichtung sorgt dafür, dass immer neue Flüssigkeit an die Elektroden herangeführt wird und an den Elektroden eine bestimmte Flïssigkeitsgeschwindigkeit herrscht.

Der zwischen den Elektroden fliessende Strom beträgt etwa $160 \mu \mathrm{A}$ bei sauerstoffgesättigtem Wasser und ist beinahe unabhängig von der Temperatur. Da man aber in der Praxis meistens den Sauerstoffgehalt nicht in Prozent der Sättigung, sondern in $\mathrm{mgO}_{2} / 1 \mathrm{zu}$ erfahren wünscht, ist eine Temperaturkompensation mit Hilfe eines eingebauten Thermistors notwendig, dessen negative Widerstandstemperaturcharakteristik die gewünschte direkte, elektrische Kompensierung erlaubt. Der verbleibende Strom von etwa $80 \mu \mathrm{A}$ ist stark genug, um auch ohne Verstärker angezeigt oder registriert $\mathrm{zu}$ werden.

Ein weiterer Thermistor dient der gleichzeitigen Temperaturmessung für eine separate Temperaturmessung oder Registrierung. 


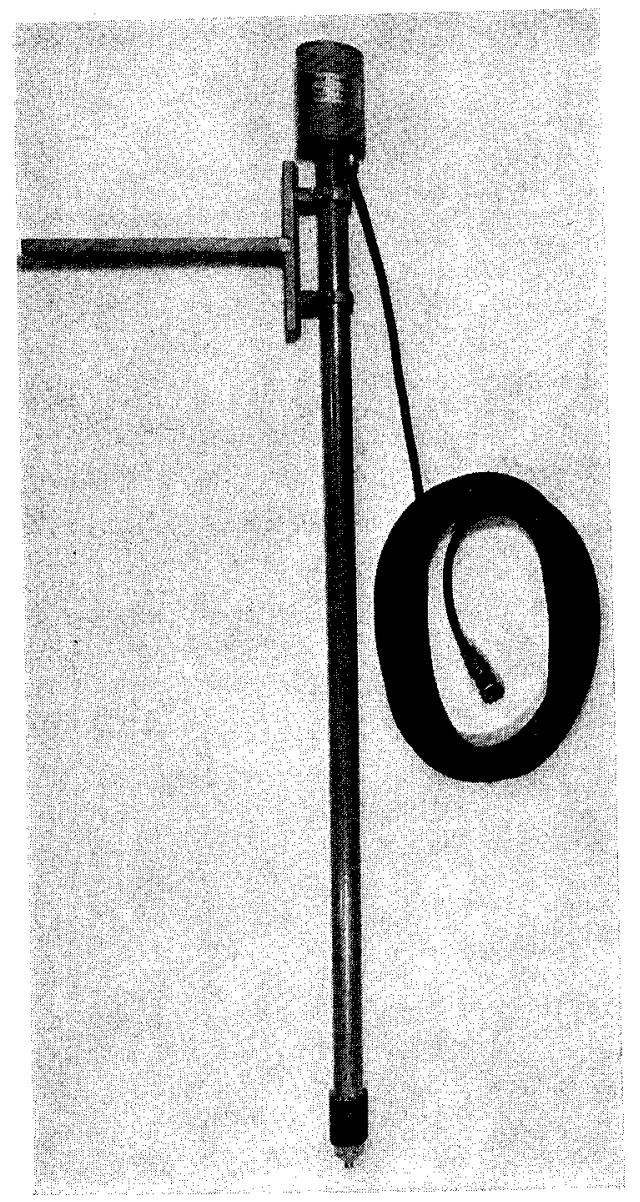

Abb. 1. Ansicht der Sauerstoffsonde.

\section{Vorteile des Systems}

4.1 Da keine Membrane, kein Elektrolyt und keine sich verändernden Elektrodenflächen da sind, kann die Sauerstoffsonde wegen der selbsttätigen Reinigungsvorrichtung auch im Belebtschlamm praktisch ohne Unterhalt und Nacheichung betrieben werden.

4.2 Die Messung ist praktisch unabhängig von der Leitfähigkeit, sobald sie nicht unter etwa $100 \mu$ Siemens/cm sinkt; sie ist unbeeinflusst vom $\mathrm{pH}-$ Wert, sobald der Elektrolyt nicht saurer ist als 4,2 (Abb. 2, 3). Freies Chlor beeinflusst die Messung merklich erst ab $5 \mathrm{mg} / \mathrm{l}$, während die zulässigen Grenzen für freies Chlor in Trinkund Badewasser unter $1 \mathrm{mg} \mathrm{Cl} \mathrm{Cl}_{2} / 1$ liegen (Tab. 1 ).

4.3 Der Meßstrom ist so stark, dass er mit einfachen Drehspulinstrumenten angezeigt oder registriert werden kann. Ein Verstärker, bei dem in längeren Zeit- 
räumen Änderungen des Verstärkungsfaktors und des Nullpunktes auftreten können, wird deshalb unnötig.

4.4 Unabhängigkeit von der Fliessgeschwindigkeit, daher Messmöglichkeit auch im stillstehenden Gewässer oder Klärbecken ohne Rühren.

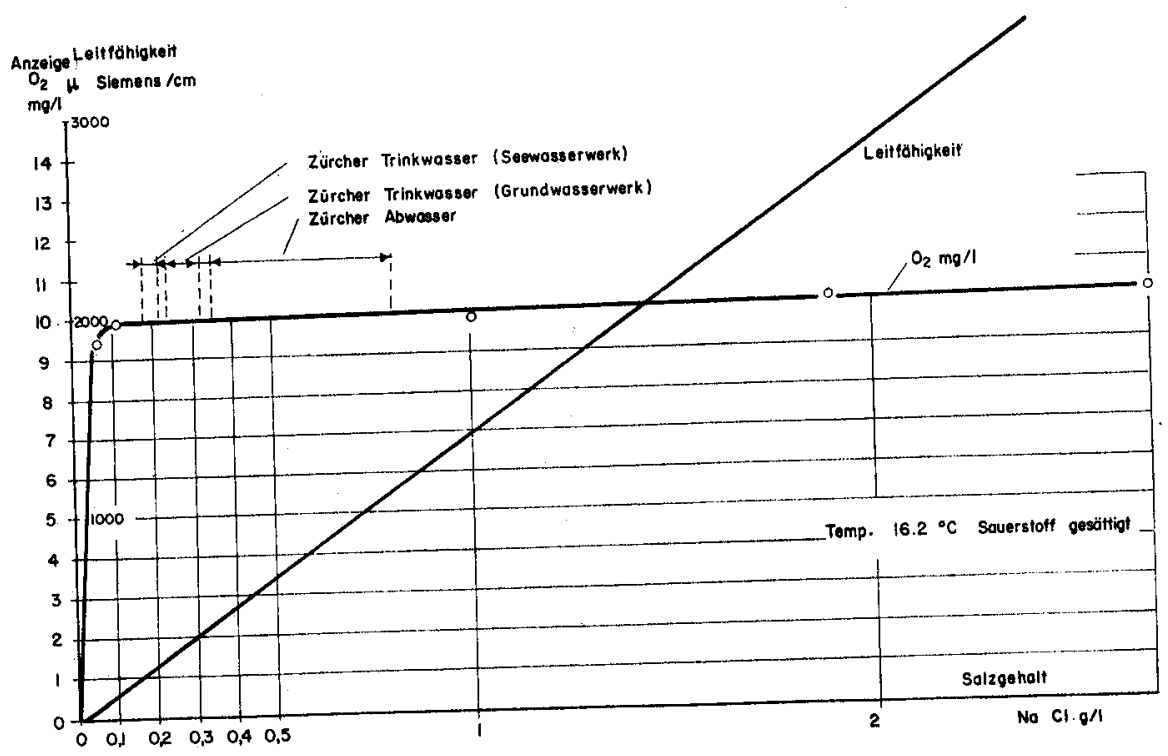

Abb. 2. Einfluss des Salzgehaltes bei sauerstoffgesättigtem Wasser.

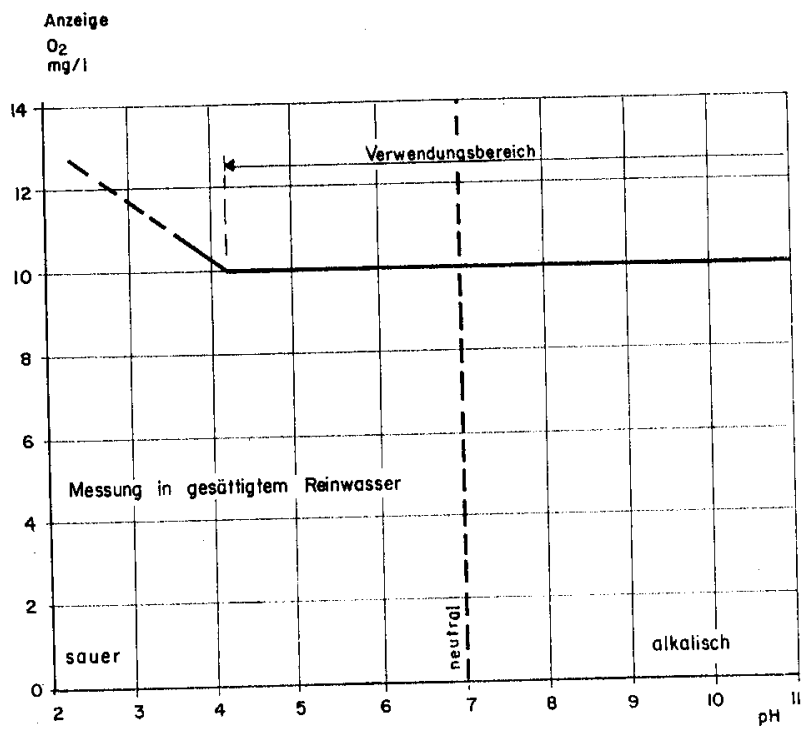

Abb. 3. Einfluss des $\mathrm{pH}$-Wertes. 
Tab. 1. Einfluss von freiem Chlor und von Eisen- und Manganchlorid.

a) Störung durch freies Chlor

Leitungswasser mit Javelle-Wasser gemischt

Freies Chlor

$\mathrm{mg} / 1$

Anzeige Sauerstoff

$\mathrm{mg} / 1$

0

9,0

6

9,5

12

10,2

18

10,6

$\left(\mathrm{O}_{2}\right.$-Gehalt konstant gehalten)

Übliche Chlordosierung im
Schwimmbecken
$\sim 0,5 \mathrm{mg}$
$\mathrm{Cl}_{2} / 1$
Trinkwasser
$0,1-0,8 \mathrm{mg}$
$\mathrm{Cl}_{2} / 1$

b) Störung durch Eisenchlovid und Manganchlorid

Leitungswasser mit $\mathrm{FeCl}_{3}$ :

$\mathrm{mg} \mathrm{FeCl}_{3} / 1$

Anzeige Sauerstoff $\mathrm{mg} / \mathrm{l}$

$0 \quad 250 \quad 500$

Leitungswasser mit $\mathrm{MnCl}_{2}$ :

$\mathrm{mg} \mathrm{MnCl}_{2} / 1$

Anzeige Sauerstoff $\mathrm{mg} / \mathrm{l}$

$\begin{array}{lrc}0 & 250 & 500 \\ 9,5 & 9,7 & 10,0\end{array}$

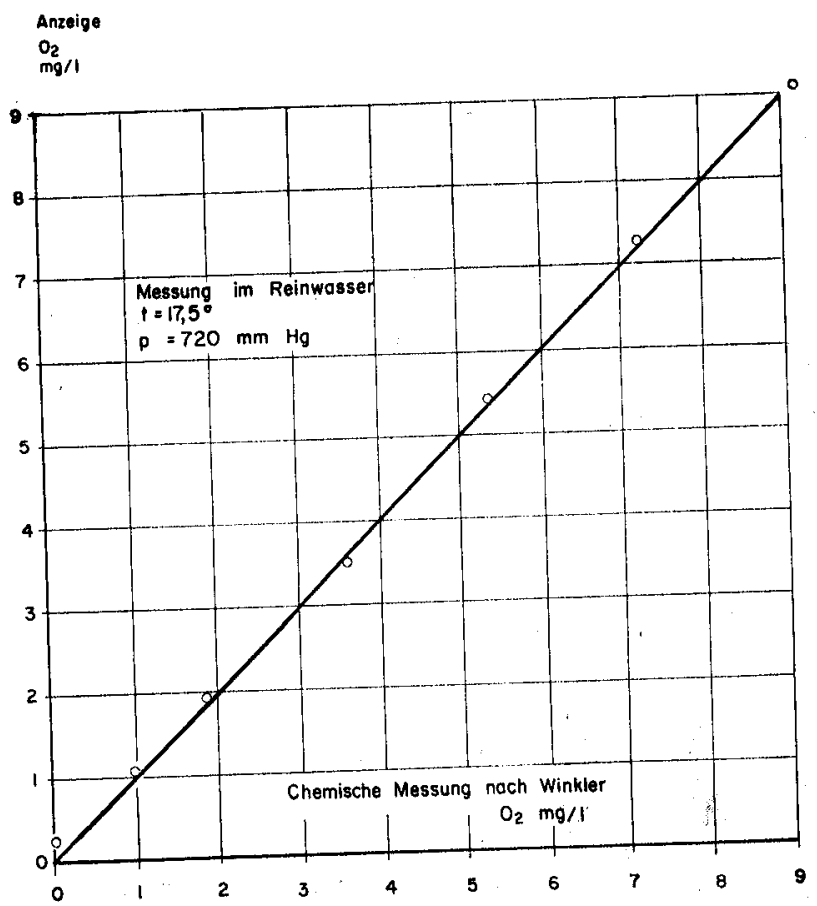

Abb. 4. Eichkurve, aufgenommen in reinem Wasser.

\section{Praktische Messungen in Kläranlagen}

Praktische Messungen wurden bisher in der Versuchsanstalt Tüffenwies der EAWAG und in den Kläranlagen Ergolz II (Baselland) und Horgen ausgeführt. Im folgenden wird nur über die Messungen in Horgen berichtet. 


\subsection{Allgemeines}

Die Messungen in der Kläranlage Horgen, die von Dezember 1967 bis April 1968 dauerten, dienten dazu, Erfahrungen unter winterlichen Verhältnissen zu sammeln und den Sauerstoffhaushalt der Kläranlage kennenzulernen. Die Messungen erfolgten mit drei Sonden gleichzeitig in verschiedenen Becken mit Hilfe eines gemeinsamen Registrierapparates. Es kann festgestellt werden, dass die herrschenden tiefen Temperaturen und die starke Vereisung keinen nachteiligen Einfluss auf die Sonden hatten.

Im Nachklärbecken sank der Sauerstoffgehalt praktisch auf Null, sobald im Belüftungsbecken nur noch etwa $2 \mathrm{mg} / \mathrm{l}$ vorhanden waren. Im Auslauf erfolgte dann bei den Zackenüberläufen wieder eine Nachbelüftung von etwa $1-2 \mathrm{mg} \mathrm{O}_{2} / 1$.

\subsection{Belüftungsbecken}

Bei den Belüftungsbecken handelt es sich um zwei nacheinander geschaltete Becken mit Inkabelüftung (Abb. 5, 6).

Der dauernde Einsatz der beiden Sonden mit Registrierung hat gezeigt, dass nach der ursprünglichen Betriebsweise mit zeitlich festgelegtem Ein- und Ausschalten eines Ventilators (ein Ventilator war dauernd in Betrieb) in den Abendstunden oft zu wenig Sauerstoff im Belüftungsbecken vorhanden war, während am Vormittag zu stark belüftet wurde. Auf Grund dieser Messungen ist man auf einen Betrieb mit zwei dauernd eingeschalteten Ventilatoren übergegangen, so dass es nur sehr selten zu Sauerstoffmangel kommt. Allerdings wird bei dieser Betriebsweise ein meistens unnötig hoher Sauerstoffgehalt aufrechterhalten.

Um einen wirtschaftlicheren Betrieb zu erreichen, müsste die Anlage mit einer von der Sauerstoffsonde automatisch geregelten Belïftung versehen werden.

Ferner wurde mit Hilfe der Sonden festgestellt, dass bei der vorliegenden Serieschaltung der beiden Becken im ersten Becken ein zu geringer, im zweiten dagegen ein $\mathrm{zu}$ hoher Sauerstoffgehalt herrschte.

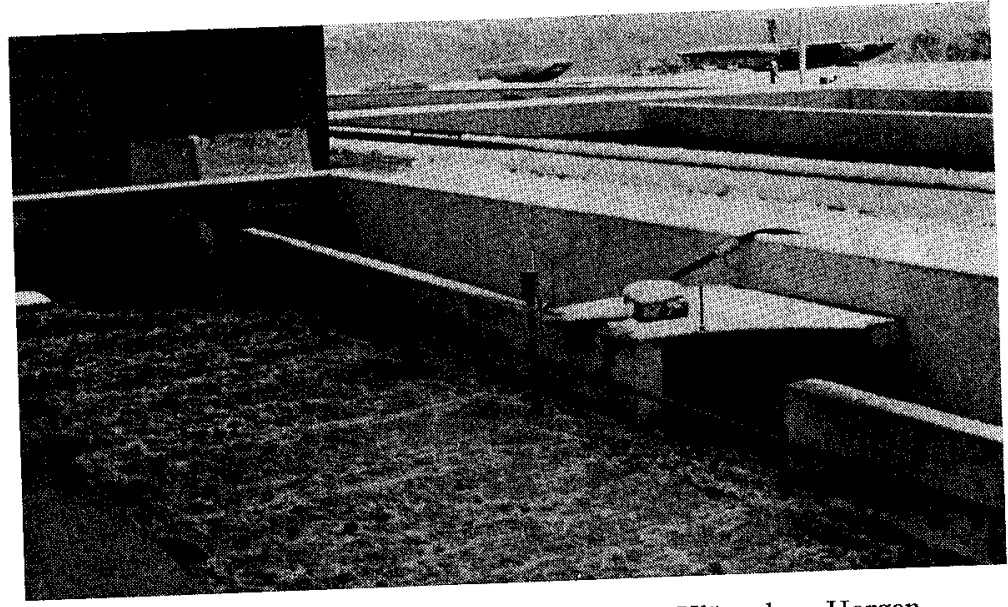

Abb. 5. Eingebaute Sauerstoffsonde in der Kläranlage Horgen 


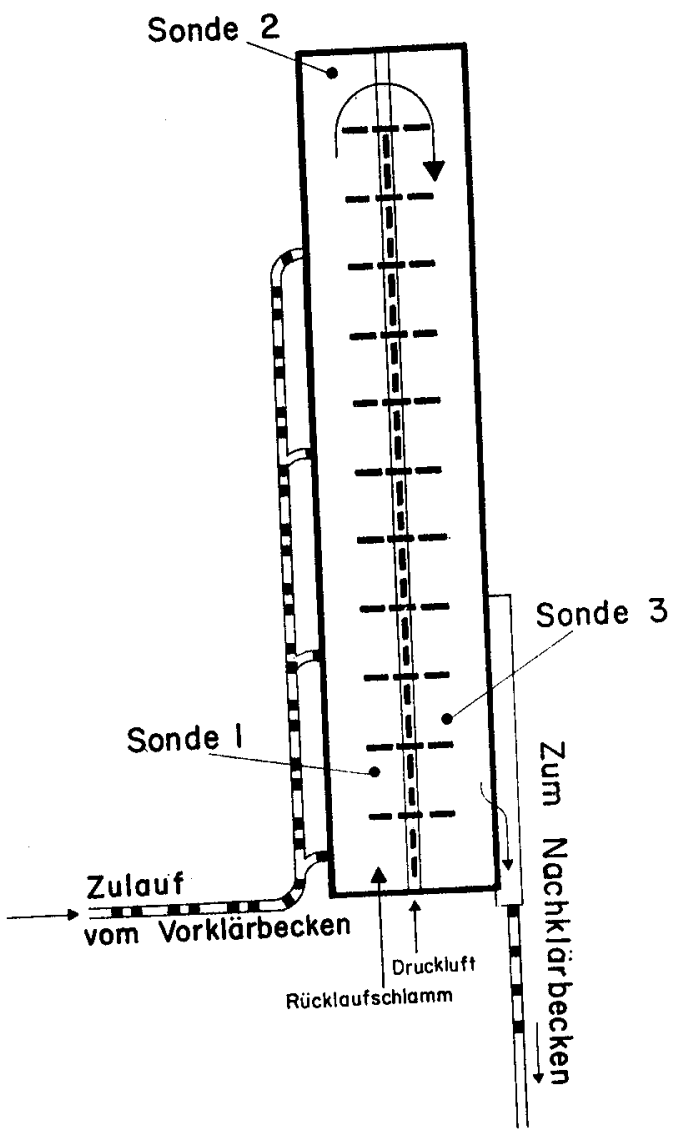

Abb. 6. Belüftungsbecken der Kläranlage Horgen mit Angaben der Meßstellen.

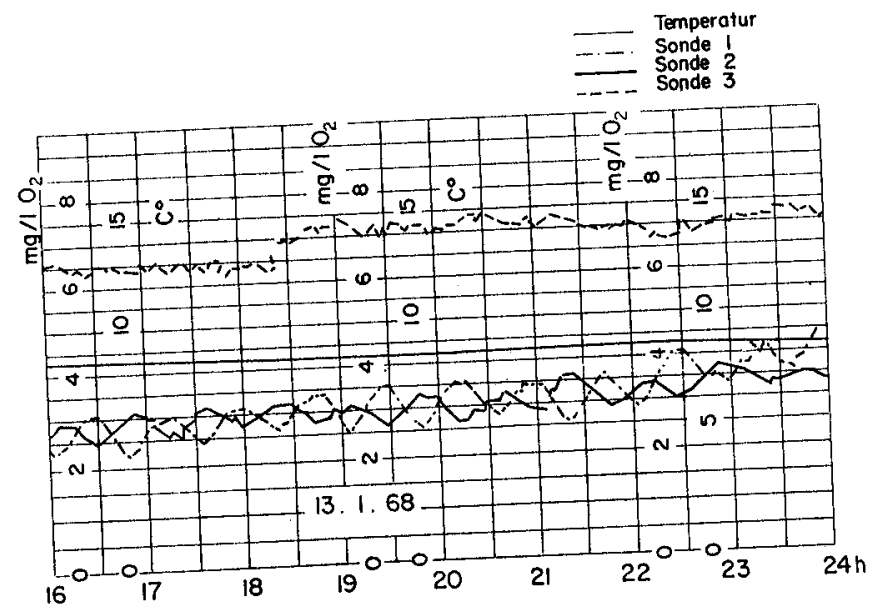

erlauf des Sauerstoffgehaltes und der Temperatur bei Belüftung mit sämtlichen Rosten. 


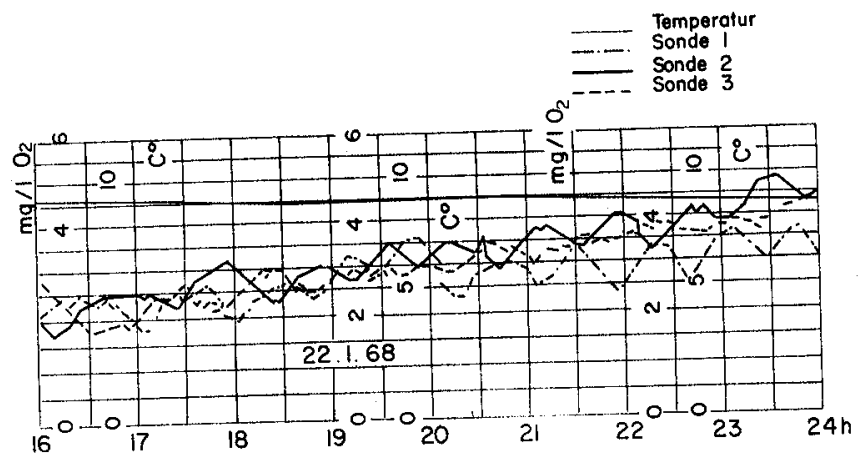

Abb. 8. Verlauf des Sauerstoffgehaltes und der Temperatur bei Belüftung mit reduzierter Rostzahl.

Um eine optimale Ausnutzung der Anlage zu erreichen, wurden im Januar 1968 im zweiten Becken von den 40 vorhandenen Belüftungsrosten 10 ausgebaut. Damit wurde die Belüftung im ersten Becken auf $114 \%$ verstärkt und im zweiten auf $85 \%$ reduziert. Gleichzeitig wurden auch Strömungsmessungen durchgeführt, welche zeigten, dass diese Massnahme keine Schlammablagerungen verursachte.

Diese Veränderungen haben sich als günstig erwiesen, wie Abb. 7 und 8 zeigen. Die periodischen Schwankungen des Sauerstoffgehaltes werden von einem Abwasserpumpwerk hervorgerufen.

\section{Regelung des Sauerstoffeintrages im Belüftungsbecken}

In einer Kläranlage ist die richtige Dosierung der Belüftung eine wichtige Aufgabe. Wie u.a. auch die Untersuchungen von WuHRMANN [3] zeigen, genügt für den biologischen Abbau im Belüftungsbecken ein Sauerstoffgehalt von etwa $1-2 \mathrm{mg} \mathrm{O} / 1$. Ein höherer Sauerstoffgehalt verursacht nur unnötige Energiekosten, erhöhte Schaumbildung und evtl. sogar einen schlechteren Abfluss. Die mögliche Senkung der Stromkosten ist je nach Anlage und Betriebsführung verschieden, doch kann man schätzen, dass in günstigen Fällen durch die Einführung der automatischen Regelung grössenordnungsmässig die Hälfte der Belüftungsstromkosten eingespart werden kann.

\subsection{Kontinuierliche Regelung}

In regeltechnischer Hinsicht wäre die kontinuierliche Regelung einer kaskadenförmigen Regelung vorzuziehen. Kontinuierlich regelbar sind z. B. die Oberflächenbelüfter mit einstellbarer Eintauchtiefe, sei es, dass das Belüftungsaggregat von einem Hubmotor gehoben und gesenkt wird, sei es, dass eine Uberfallkante mit einem Servomotor verstellt wird.

Die sauberste Lösung wäre hierbei die Verwendung eines Proportionalreglers mit einstellbarem Sollwert und Proportionalitätsband. Das erfordert jedoch eine Rückführung und einen elektronischen Regler und Verstärker, die ziemlich aufwendig sind. Da aber die erforderliche Regelgenauigkeit nicht sehr gross, bzw. die Totzeit von etwa 5-8 Minuten verhältnismässig kurz ist, würde auch ein preiswertes Zeigerinstrument oder ein Fallbügelschreiber mit Maximum- und Minimum-Sollwertkon- 
takten genügen. In diesem Falle würde bei Nichtübereinstimmung mit dem Sollwert der Servomotor die Verstellung entweder in die eine oder in die andere Richtung betätigen, was dem Wesen nach einer Integralregelung entspricht.

\subsection{Kaskadenförmige Regelung}

In vielen Fällen hat man mit Anlagen zu tun, die nur kaskadenförmig, also in 2-3 Stufen gesteuert werden können. Dabei handelt es sich z. B. um einzeln zuschaltbare Ventilatoren einer Ventilatorgruppe oder um Oberflächenbelüfter mit stufenweise schaltbaren Drehzahlen. Hierbei muss das Sondensignal in bestimmten Zeitintervallen abgefragt werden, und bei Nichtübereinstimmung mit dem Sollwert werden die Motorschützen auf die nächste Stufe hinauf- oder hinuntergeschaltet. Damit es nicht zu einem unstabilen Betrieb kommt, müssen die Zeitintervalle richtig angepasst werden.

\section{ZUSAMMENFASSUNG}

Es wurde eine neue, membranlose Sauerstoffsonde entwickelt, die auch im Belebtschlamm weder Unterhalt noch Nacheichung erfordert und deren Anzeige vom Elektrolytgehalt und $\mathrm{pH}$ Wert praktisch unbeeinflusst bleibt.

Im weiteren wird über Erfahrungen, die im praktischen Einsatz des Gerätes in der Kläranlage Horgen gewonnen wurden, berichtet.

Abschliessend werden die Möglichkeiten der Regelung des Sauerstoffeintrages im Belüftungsbecken mit verschiedenen Systemen kurz erörtert.

\section{SUMMARY}

A newly developed apparatus for measuring $\mathrm{O}_{2}$ concentrations is described. The apparatus works without any membrane, and no maintenance and readjustment is necessary even if it is used in activated sludge. The readings are practically not influenced by salt concentrations and $\mathrm{pH}$ of the medium.

Practical experience with the use of the apparatus at the Horgen sewage treatment plant is reported. The application of the apparatus for continuous control of oxygen transfer in aeration basins using different aeration systems is mentioned.

\section{LITERATURVERZEICHNIS}

[1] Tö̀t F., Elektrochemische Sauerstoffmessungen (Verlag de Gruyter, Berlin 1958).

[2] Амв ÜHL H., Die praktische Anwendung der elektrochemischen Sauerstoffbestimmung im Wasser. II. Kurzzeitmessung der Oxytestev, Schweiz. Z. Hydrol. 20, 341-359 (1958).

[3] Wuhrmann K., Grundlagen für die Dimensionierung der Belïftung bei Belebtschlammanlagen, Schweiz. Z. Hydrol. 26, 310-337 (1964).

Adresse des Autors: L. Kalman, dipl. Ing., Rautihalde 10, 8048 Zürich.

Separatdruck aus der Schweiz. Zeitschrift für Hydrologie 31, Fasc. 1 (1969) Herausgegeben mit Unterstützung der Stiftung der Wirtschaft zur Förderung des Gewässerschutzes in der Schweiz 